\title{
Os padrões de distribuição dos peixes de uma laguna costeira aberta no Atlântico oeste subtropical são influenciados pelas variações espaciais e sazonais?
}

\author{
Dafne Borgo 1 \\ André Pereira Catani ${ }^{3}$ \\ Gisela Costa Ribeiro ${ }^{2}$ \\ Helen Audrey Pichler ${ }^{3}$ \\ Mauricio Hostim-Silva ${ }^{4}$ \\ Leandro Clezar ${ }^{2}$ \\ Lilyane de Oliveira Santos ${ }^{1 *}$ \\ Henry Louis Spach ${ }^{1}$ \\ ${ }^{1}$ Universidade Federal do Paraná, Centro de Estudos do Mar \\ Pós-graduação em Sistemas Costeiros e Oceânicos, CEP 83.255-976, Pontal do Paraná - PR, Brasil \\ ${ }^{2}$ Universidade Federal de Santa Catarina, Núcleo de Estudos do Mar, Florianópolis - SC, Brasil \\ ${ }^{3}$ Universidade Federal do Paraná, Setor de Ciências Biológicas \\ Pós-graduação em Ecologia e Conservação, Curitiba - PR, Brasil \\ ${ }^{4}$ Universidade Federal do Espirito Santo \\ Pós-graduação em Biodiversidade Tropical, São Mateus - ES, Brasil \\ * Autor para correspondência \\ lilyaneoliveirasantos@gmail.com
}

Submetido em 17/09/2014

Aceito para publicação em 23/06/2015

\section{Resumo}

Este estudo teve como objetivo descrever as variações espaço-temporais da ictiofauna em uma laguna costeira aberta. Os peixes foram coletados na Lagoa da Conceição, Florianópolis-SC, totalizando 19 meses de amostragem entre outubro de 1987 e novembro de 1989. Foram realizadas coletas em sete pontos, sendo que em cada um foram empregados lances de tarrafa de diferentes malhas, rede de mão do tipo puçá, rede de arrasto de praia e pesca manual com anzóis. Foram coletados 8.299 indivíduos, classificados em 61 espécies pertencentes a 28 famílias de peixes, totalizando $52.902,4 \mathrm{~g}$. Em relação à permanência na laguna, 48 espécies $(80 \%)$ foram classificadas como visitantes e as outras 12 eram residentes. Foram observadas diferenças significativas entre as estações (n, biomassa, riqueza de espécies e H’Loge), entre as áreas (n e riqueza de espécies) e nas interações entre os fatores estação e área (n e biomassa). Os dados sobre a ictiofauna reforçam a importância ecológica do ambiente da laguna costeira como área berçário e de alimentação, já que a maioria das espécies encontra-se em fases de recrutamento dentro da Lagoa da Conceição.

Palavras-chave: Ictiofauna; Laguna; Variação espaço-temporal 


\section{Abstract}

Are the fish distribution patterns in an open coastal lagoon in the subtropical west Atlantic influenced by spatial and seasonal variations? This study aimed to describe the spatial-temporal variations of the fish fauna in an open coastal lagoon. Fish were collected in Lagoa da Conceição, Florianópolis, Santa Catarina, Brazil, totaling 19 sampling months between October 1987 and November 1989. Sampling took place at 7 sites, and in each of them cast net throws of different meshes, "puçá" handheld fishing net, beach seine fishing net, and manual fishing hooks were used. A total of 8,299 individuals were collected, classified into 61 species belonging to 28 fish families, totaling 52,902.4 g. Regarding stay in the lagoon, 48 species (80\%) were classified as visitors and the other 12 were residents. Significant differences were observed between seasons (n, biomass, species richness, and H'Loge), between areas ( $\mathrm{n}$ and species richness), and in the interactions between the factors season and area (n and biomass). Data on fish fauna reinforce the ecological significance of the coastal lagoon environment as a nursery and feeding area, since most species are at recruitment stages within Lagoa da Conceição.

Key words: Fish fauna; Lagoon; Spatial-temporal variation

\section{Introdução}

As áreas costeiras possuem elevada importância para a biota aquática. Juntamente com a alta produtividade, a presença de áreas rasas propicia uma variedade de habitats que suportam diversas espécies de organismos em vários estágios do ciclo de vida (YAÑEZ-ARANCIBIA, 1986). Por serem ambientes protegidos, atuam como refúgios seguros, locais de alimentação e crescimento para inúmeras espécies da biota aquática, em especial para a ictiofauna (BLABER; BLABER, 1980; ELLIOTT; MCLUSKY, 2002). A alta produtividade primária e secundária dessas áreas tem grande influência sobre a densidade, riqueza e biomassa de peixes (DAY et al., 1989; WHITFIELD, 1999).

Os ambientes lagunares representam 13\% da zona costeira mundial e, juntamente com as demais áreas costeiras, contribuem com uma grande parcela da riqueza ecológica da biosfera (KNOPPERS, 1994; CONSTANZA et al., 1997). Na América do Sul, cerca de $12,2 \%$ da extensão da costa se apresenta sob forma lagunar, o que representa $10,3 \%$ da extensão mundial ocupada por lagoas costeiras (CROMWELL, 1971).

Além de fornecerem recursos alimentares, as lagoas costeiras proporcionam locais de criação e proteção contra a predação de juvenis (KJERFVE, 1994). Nestes ambientes, a fauna é altamente dinâmica devido às interações intra e interespecíficas e as limitações fisiológicas e de estratégia de vida (KENNISH; PEARL, 2010). As variações espaciais e temporais dos parâmetros abióticos, tais como salindade e temperatura, afetam diretamente a biota aquática destes ambientes. Para os peixes, tais variações têm importante papel na estruturação das assembleias (JAUREGUIZAR et al., 2004; AKIN et al., 2005; BARLETTA et al., 2008).

Uma característica relevante das lagunas costeiras é a ausência de zonação biótica e abiótica na coluna d'água devido às baixas profundidades, o que facilita a homogeneização vertical da coluna d'água pela ação dos ventos (KJERVFE, 1994; SUZUKI et al., 1998). Assim, observam-se padrões de distribuição horizontal da diversidade de peixes, cujas espécies podem ser encontradas em todo o corpo das lagunas costeiras (FROTA; CARAMASCHI, 1998; LIMA et al., 2001; MARQUES, 2005). Em escala local, esses padrões são influenciados pela salinidade, temperatura, oxigênio dissolvido e transparência da água (KJERVFE, 1994).

Em escala temporal, os peixes são influenciados por variações circadianas ou nictimerais até variações interanuais (MACI; BASSET, 2009). Processos ecológicos relacionados ao recrutamento e à reprodução são diretamente afetados pelas variações temporais (GREENWOOD; HILL, 2003; JAUREGUIZAR et al., 2004).

Estudos realizados em lagoas costeiras, de regiões subtropicais e temperadas, corroboram a influência das escalas espaciais e temporais nos padrões e distribuição dos peixes (PÉREZ-RUZAFA et al., 2006; VERDIELLCUBEDO et al., 2006; AMEZCUA; AMEZCUA- 
LINARES, 2014). Um padrão similar foi observado nas regiões sudeste e sul do Brasil (ANDREATA et al., 1989; SIERRA DE LEDO et al., 1993; LOEBMANN; VIEIRA, 2005).

A Lagoa da Conceição, classificada como uma laguna costeira está localizada em uma ilha costeira próxima a um mosaico de ecossistemas, como estuários, lagoas, manguezais e praias arenosas (SIERRA DE LEDO, 1997). É uma lagoa conhecida por ser uma das maiores atrações turísticas e de lazer da Ilha de Santa Catarina (recreação infanto-juvenil, esportes, cultura, turismo, comércio, gastronomia), tanto na alta como na baixa temporada. Por estas razões, ela é considerada o ambiente de maior potencial natural, turístico e econômico da Ilha de Santa Catarina (SIERRA DE LEDO et al., 1985). Portanto, possuir o conhecimento sobre a composição da ictiofauna e como ela varia tanto no espaço como no tempo é fundamental para um planejamento das formas de ocupação do seu entorno e de uso das espécies de peixes, seja pela pesca comercial ou recreativa (KUPSCHUS; TREMAIN, 2001). Desta forma, este trabalho teve como objetivo testar a hipótese de que a estrutura da comunidade de peixes em uma laguna costeira aberta no Atlântico oeste subtropical (sul do Brasil) é diferente espacialmente e temporalmente.

\section{Material e Métodos}

\section{Área de estudo}

A área de estudo é a Lagoa da Conceição, uma laguna costeira aberta localizada no centro-leste da Ilha de Santa Catarina entre $27^{\circ} 22^{\prime}$ e $27^{\circ} 51$ 'S, e $48^{\circ} 20^{\prime}$ e 48 37'O (Figura 1). A Lagoa da Conceição tem um comprimento total (eixo norte-sul) de 13,5 km, uma superfície de $19,2 \mathrm{~km}^{2}$, largura entre 2,5 e $0,15 \mathrm{~km}$ e profundidade que varia de $0,5 \mathrm{~m}$ nos bancos de areia a 8,8 m nos canais (MUEHE; GOMES JR., 1999; LISBOA et al., 2008). Está direcionada paralela e contígua a margem continental e possui forma alongada e orientada na direção N-NE/S-SW, sendo que sua bacia hidrográfica ocupa uma área de $431 \mathrm{~km}^{2}$, rodeada por $180 \mathrm{~km}$ de costa, no limite da região subtropical (KLINGEBIEL; SIERRA DE LEDO, 1997). A lagoa mantém uma ligação

FIGURA 1: Compartimentos da Lagoa da Conceição quanto à salinidade e localização dos sete pontos de amostragem onde as coletas foram realizadas.

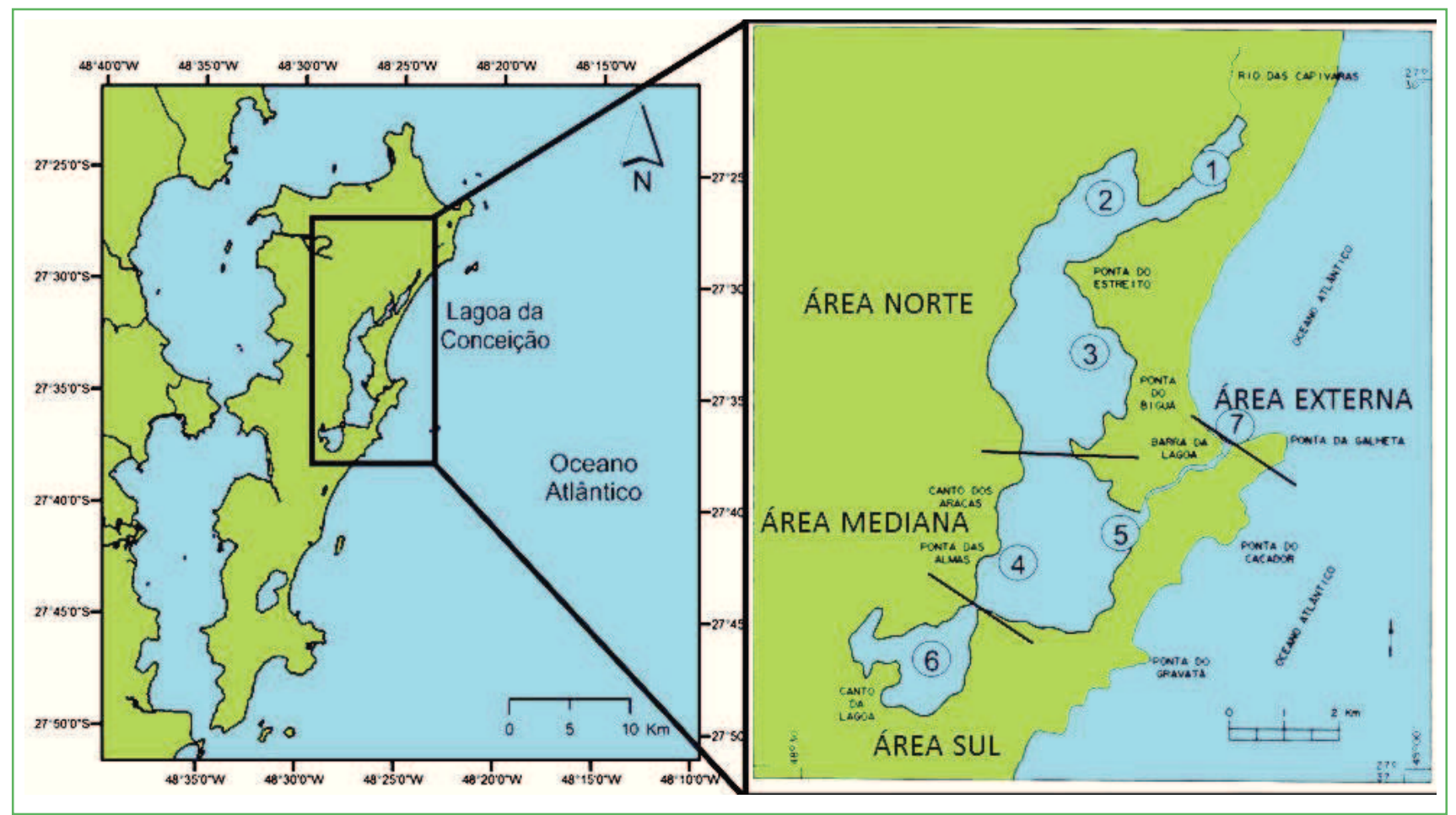


com o mar através do Canal da Barra, que desde 1982 fica permanentemente aberto, influenciando diretamente na salinidade da água (LISBOA et al., 2008).

Knoppers et al. (1984) e Odebrecht e Caruso Jr. (1987) setorizaram e caracterizaram a Lagoa da Conceição em três regiões distintas de acordo com as variáveis físicas e químicas: Região Norte (Área N neste trabalho), sofre a maior influência fluvial, proveniente do rio João Gualberto e a salinidade média é de 11,0 ups; Região Central (Área M): inclui o canal de acesso ao mar aberto, cuja coluna d'água apresenta salinidades médias de 18,5 ups; Região Sul (Área S), a mais isolada, pois apresenta uma ponte com abertura de $3 \mathrm{~m}$ de largura na sua conexão com a região central, a qual estrangula o canal natural. A predominância é de águas com salinidade média de 6,7 ups. Neste trabalho foi ainda amostrada uma área (área 7) que apresenta características marcadamente similares ao ambiente marinho devido a sua proximidade com o mar (salinidade 35 ups), uma vez que está localizada na área externa próxima da entrada do canal da barra.

O clima da região, segundo a classificação de Köppen, é subtropical úmido ( $\mathrm{Cfa}$ ), cujo regime de chuvas apresenta uma sazonalidade bem definida, com período chuvoso nos meses da primavera e verão (setembro a março), com média mensal de $149 \mathrm{~mm}$, e período mais seco nos meses de outono e inverno (abril a agosto), com média mensal de 95 mm (MPB, 2011).

A circulação das águas tem um regime condicionado pelas marés, ventos e ciclos de evaporação-precipitação em sua bacia hidrográfica. A variabilidade nos valores de salinidade está condicionada principalmente aos ciclos de evaporação-precipitação (ODEBRECHT, CARUSO JR., 1987). Assim, em períodos em que coincidiram altas taxas de evaporação e pouca chuva, as salinidades em toda a lagoa foram as mais altas de todo o período.

\section{Delineamento amostral}

Para se conhecer os padrões de distribuição dos peixes na Lagoa da Conceição, foram utilizadas diferentes estratégias amostrais. Foram realizadas amostragens em sete pontos de coleta (Figura 1), nestes foram empregados, mensalmente, igual número de lances de tarrafa de diferentes malhas, rede de mão do tipo puçá, rede de arrasto de praia e pesca manual com anzois.

O material biológico coletado foi acondicionado em sacos plásticos, etiquetados e transportados ao laboratório. Cada espécie coletada foi identificada segundo Figueiredo e Menezes $(1978 ; 1980 ; 2000)$ e Menezes e Figueiredo (1980) e, em seguida os peixes foram quantificados, medidos (mm) e pesados (g). As espécies de peixe foram classificadas utilizando a base virtual de dados fishbase (FROESE; PAULY, 2014), e posteriormente foram enquadradas de acordo com Vieira e Musick (1993) em residentes e visitantes.

\section{Tratamento dos dados}

Para descrever composição e estrutura da ictiofauna, bem como para as análises das variações espaço-temporais dos descritores da comunidade como número de indivíduos (n), biomassa (em g), riqueza de espécies, diversidade de Shannon-Wiener $\left(\mathrm{H}^{\prime} \log _{\mathrm{e}}\right)$ e equitatividade de Pielou (J'), foram utilizados dados coletados durante 19 meses entre outubro de 1987 e novembro de 1989.

Com o intuito de facilitar o tratamento dos dados, os meses foram agrupados em estações sendo Verão (Ver, meses de janeiro a março), Outono (Out, abril a junho), Inverno (Inv, julho a setembro) e Primavera (Prim, outubro a dezembro). Para as análises dos descritores, foram retirados todos os indivíduos que não tiveram sua espécie identificada (Eucinostomus sp., Lutjanus sp., Mycteroperca sp. e Strongylura sp.) com exceção de Paralichthys sp. e Poecilia sp. que apresentaram apenas um indivíduo destes gêneros. Por terem representado mais de $50 \%$ dos peixes capturados, as espécies da família Mugilidae foram removidas de todas as análises para evitar ruído, sendo que as variações espaçotemporais em $\mathrm{n}$ e biomassa foram descritas a parte.

Para se testar a significância das diferenças nos descritores da assembleia, inicialmente os dados de $\mathrm{n}$ e biomassa foram transformados em $\log (\mathrm{x}+1)$, enquanto os demais descritores como riqueza de espécies, $\mathrm{H}^{\prime} \log _{\mathrm{e}}$ e J', foram utilizados os dados sem transformações. 
Tendo em vista o diferente número de amostra em cada estação do ano e área, os dados foram submetidos à rotina PERMANOVA (análise de variância permutacional multivariada) com 9999 permutações, sendo os fatores estação e área (teste global). Quando diferenças significativas foram observadas, os dados foram submetidos ao teste a posteriori (teste t pareado) da mesma rotina, testando-se a diferença entre as estações do ano em cada área (ANDERSON et al., 2008).

Para visualizar graficamente as diferenças encontradas na PERMANOVA entre as áreas e entre as estações do ano, foi realizada a análise canônica de coordenadas principais (CAP) (ANDERSON et al., 2008) com os dados de abundância (N) transformados em $\log (\mathrm{x}+1)$. Os agrupamentos foram gerados com 9999 permutações. Dentro da análise CAP, correlações de Spearman foram utilizadas para determinar quais espécies (vetores) foram responsáveis pelos agrupamentos. O nível de correlação utilizado para selecionar as espécies foi acima de $0,5(\rho>0,5)$.

\section{Resultados}

\section{Composição e estrutura}

Foram coletados 8298 indivíduos, identificados em 61 espécies, pertencentes a 28 famílias de peixes. A família Carangidae apresentou o maior número de espécies (7) seguidas por Engraulidae e Gerreidae (6) e ainda Clupeidae, Gobiidae e Sciaenidae com 5 cada. A assembleia foi numericamente dominada por poucas espécies, sendo que apenas Mugil curema (20,8\%), Mugil spp. (17,8\%), Mugil liza (13,9\%), Atherinella brasiliensis (13,6\%) e Eucinostomus argenteus (7,3\%) representaram aproximadamente $73 \%$ do total amostrado e 32 espécies representaram $0,1 \%$ ou menos do total (Tabela 1 ).

TABELA 1: Composição específica, abundância total (n) e relativa (\%), biomassa total e relativa (\%), e guilda de permanência ( $\mathrm{R}$ = residente; $\mathrm{V}$ = visitante) dos peixes coletados na Lagoa da Conceição, $\mathrm{SC}$, durante os anos de 1987, 1988 e 1989; os números sobrescritos indicam a referência bibliográfica das guildas de permanência ( ${ }^{1}$ CHAVES; VENDEL, 1997; ${ }^{2}$ MATSUURA, 1998; ${ }^{3} \mathrm{CHAVES}$; OTTO, 1998; ${ }^{4}$ FIGUEIREDO; MENEZES, 2000; ${ }^{5}$ GARCIA; VIEIRA 2001; ${ }^{6}$ VENDEL et al., 2010; ${ }^{7}$ VILAR et al., 2011; ${ }^{8}$ PASSOS et al., 2013; ${ }^{9}$ FROESE; PAULY, 2014).

\begin{tabular}{|c|c|c|c|c|c|}
\hline \multirow{2}{*}{ Famílias/Espécies } & \multicolumn{2}{|c|}{$\mathbf{n}$} & \multicolumn{2}{|c|}{ Biomassa (g) } & \multirow{2}{*}{$\begin{array}{c}\text { Guildas de } \\
\text { permanência }\end{array}$} \\
\hline & total & $\%$ & total & $\%$ & \\
\hline \multicolumn{6}{|l|}{ Achiridae } \\
\hline Achirus lineatus & 3 & $<0,1$ & 22,9 & $<0,1$ & $\mathrm{R}^{8}$ \\
\hline \multicolumn{6}{|l|}{ Ariidae } \\
\hline Genidens genidens & 29 & 0,3 & 303,3 & 0,6 & $\mathrm{~V}^{8}$ \\
\hline \multicolumn{6}{|l|}{ Atherinopsidae } \\
\hline Atherinella brasiliensis & 1128 & 13,6 & 3076,6 & 5,8 & $\mathrm{R}^{8}$ \\
\hline Odontesthes bonariensis & 5 & 0,1 & 61,7 & 0,1 & $\mathrm{R}^{8}$ \\
\hline \multicolumn{6}{|l|}{ Belonidae } \\
\hline Strongylura marina & 21 & 0,3 & 696,4 & 1,3 & $\mathrm{~V}^{8}$ \\
\hline Strongylura sp. & 2 & $<0,1$ & 7,4 & $<0,1$ & \\
\hline \multicolumn{6}{|l|}{ Blenniidae } \\
\hline Parablennius pilicornis & 1 & $<0,1$ & 0,6 & $<0,1$ & $\mathrm{~V}^{9}$ \\
\hline \multicolumn{6}{|l|}{ Bregmacerotidae } \\
\hline Bregmaceros atlanticus & 1 & $<0,1$ & 0,7 & $<0,1$ & $\mathrm{~V}^{9}$ \\
\hline \multicolumn{6}{|l|}{ Carangidae } \\
\hline Caranx hippos & 5 & 0,1 & 16,1 & $<0,1$ & $\mathrm{~V}^{8}$ \\
\hline Caranx latus & 12 & 0,1 & 83,6 & 0,2 & $\mathrm{~V}^{8}$ \\
\hline Oligoplites palometa & 2 & $<0,1$ & 1,0 & $<0,1$ & $\mathrm{~V}^{9}$ \\
\hline Oligoplites saurus & 10 & 0,1 & 145,2 & 0,3 & $\mathrm{~V}^{8}$ \\
\hline
\end{tabular}




\begin{tabular}{|c|c|c|c|c|c|}
\hline Trachinotus carolinus & 1 & $<0,1$ & 1,9 & $<0,1$ & $\mathrm{~V}^{9}$ \\
\hline Trachinotus falcatus & 19 & 0,2 & 49,1 & 0,1 & $\mathrm{~V}^{8}$ \\
\hline Trachinotus marginatus & 71 & 0,9 & 88,0 & 0,2 & $\mathrm{~V}^{8}$ \\
\hline \multicolumn{6}{|l|}{ Cichlidae } \\
\hline Geophagus brasiliensis & 8 & 0,1 & 157,4 & 0,3 & $\mathrm{~V}^{9}$ \\
\hline \multicolumn{6}{|l|}{ Clupeidae } \\
\hline Brevoortia pectinata & 3 & $<0,1$ & 14,6 & $<0,1$ & $\mathrm{~V}^{9}$ \\
\hline Harengula clupeola & 130 & 1,6 & 914,1 & 1,7 & $\mathrm{~V}^{5}$ \\
\hline Opisthonema oglinum & 10 & 0,1 & 193,6 & 0,4 & $\mathrm{~V}^{7}$ \\
\hline Sardinella brasiliensis & 23 & 0,3 & 154,0 & 0,3 & $\mathrm{~V}^{2}$ \\
\hline \multicolumn{6}{|l|}{ Dactylopteridae } \\
\hline Dactylopterus volitans & 1 & $<0,1$ & 8,5 & $<0,1$ & $\mathrm{~V}^{9}$ \\
\hline \multicolumn{6}{|l|}{ Engraulidae } \\
\hline Anchoa januaria & 1 & $<0,1$ & 2,4 & $<0,1$ & $\mathrm{R}^{8}$ \\
\hline Anchoa lyolepis & 6 & 0,1 & 11,5 & $<0,1$ & $\mathrm{R}^{8}$ \\
\hline Anchoa tricolor & 36 & 0,4 & 101,0 & 0,2 & $\mathrm{R}^{8}$ \\
\hline Cetengraulis edentulus & 44 & 0,5 & 217,8 & 0,4 & $\mathrm{~V}^{8}$ \\
\hline Engraulis anchoita & 1 & $<0,1$ & 9,5 & $<0,1$ & $\mathrm{~V}^{8}$ \\
\hline Lycengraulis grossidens & 1 & $<0,1$ & 12,0 & $<0,1$ & $\mathrm{~V}^{8}$ \\
\hline \multicolumn{6}{|l|}{ Gerreidae } \\
\hline Diapterus rhombeus & 8 & 0,1 & 4,3 & $<0,1$ & $\mathrm{~V}^{3}$ \\
\hline Eucinostomus argenteus & 603 & 7,3 & 3555,5 & 6,7 & $\mathrm{~V}^{8}$ \\
\hline Eucinostomus gula & 167 & 2,0 & 1355,8 & 2,6 & $\mathrm{~V}^{8}$ \\
\hline Eucinostomus melanopterus & 240 & 2,9 & 1625,1 & 3,1 & $\mathrm{~V}^{8}$ \\
\hline Eucinostomus sp. & 1 & $<0,1$ & 0,4 & $<0,1$ & \\
\hline Eugerres brasilianus & 74 & 0,9 & 356,2 & 0,7 & $\mathrm{~V}^{8}$ \\
\hline Ulaema lefroyi & 339 & 4,1 & 315,9 & 0,6 & $\mathrm{R}^{8}$ \\
\hline \multicolumn{6}{|l|}{ Gobiidae } \\
\hline Bathygobius soporator & 52 & 0,6 & 479,1 & 0,9 & $\mathrm{R}^{6}$ \\
\hline Ctenogobius boleosoma & 34 & 0,4 & 75,6 & 0,1 & $\mathrm{~V}^{8}$ \\
\hline Ctenogobius shufeldti & 14 & 0,2 & 18,3 & $<0,1$ & $\mathrm{~V}^{8}$ \\
\hline Ctenogobius stigmaticus & 6 & 0,1 & 7,9 & $<0,1$ & $\mathrm{R}^{8}$ \\
\hline Gobionellus oceanicus & 1 & $<0,1$ & 12,2 & $<0,1$ & $\mathrm{R}^{8}$ \\
\hline \multicolumn{6}{|l|}{ Haemulidae } \\
\hline Haemulon steindachneri & 22 & 0,3 & 521,9 & 1,0 & $\mathrm{~V}^{4}$ \\
\hline Orthopristis ruber & 33 & 0,4 & 69,6 & 0,1 & $\mathrm{~V}^{8}$ \\
\hline \multicolumn{6}{|l|}{ Hemiramphidae } \\
\hline Hemiramphus brasiliensis & 2 & $<0,1$ & 63,6 & 0,1 & $\mathrm{~V}^{8}$ \\
\hline Hyporhamphus unifasciatus & 18 & 0,2 & 165,3 & 0,3 & $\mathrm{~V}^{8}$ \\
\hline \multicolumn{6}{|l|}{ Lutjanidae } \\
\hline Lutjanus analis & 11 & 0,1 & 45,0 & 0,1 & $\mathrm{~V}^{8}$ \\
\hline \multicolumn{6}{|l|}{ Monacanthidae } \\
\hline Monacanthus ciliatus & 1 & $<0,1$ & 2,0 & $<0,1$ & $\mathrm{~V}^{9}$ \\
\hline Stephanolepis hispidus & 2 & $<0,1$ & 17,4 & $<0,1$ & $\mathrm{~V}^{9}$ \\
\hline \multicolumn{6}{|l|}{ Mugilidae } \\
\hline Mugil curema & 1725 & 20,8 & 8901,1 & 16,8 & $\mathrm{~V}^{8}$ \\
\hline Mugil gaimardianus & 13 & 0,2 & 126,7 & 0,2 & $\mathrm{~V}^{9}$ \\
\hline Mugil liza & 1154 & 13,9 & 24250,6 & 45,8 & $\mathrm{~V}^{8}$ \\
\hline Mugil spp. & 1479 & 17,8 & 583,8 & 1,1 & \\
\hline \multicolumn{6}{|l|}{ Narcinidae } \\
\hline Narcine brasiliensis & 2 & $<0,1$ & 999,3 & 1,9 & $\mathrm{~V}^{8}$ \\
\hline
\end{tabular}




\begin{tabular}{|c|c|c|c|c|c|}
\hline \multicolumn{6}{|l|}{ Ophichthidae } \\
\hline Ophichthus gomesii & 1 & $<0,1$ & 86,3 & 0,2 & $\mathrm{~V}^{8}$ \\
\hline \multicolumn{6}{|l|}{ Paralichthyidae } \\
\hline Citharichthys spilopterus & 12 & 0,1 & 220,2 & 0,4 & $\mathrm{R}^{1}$ \\
\hline Paralichthys sp. & 1 & $<0,1$ & 7,4 & $<0,1$ & \\
\hline \multicolumn{6}{|l|}{ Poeciliidae } \\
\hline Poecilia vivipara & 241 & 2,9 & 186,1 & 0,4 & \\
\hline \multicolumn{6}{|l|}{ Pomatomidae } \\
\hline Pomatomus saltatrix & 106 & 1,3 & 930,4 & 1,8 & $\mathrm{~V}^{8}$ \\
\hline \multicolumn{6}{|l|}{ Sciaenidae } \\
\hline Cynoscion leiarchus & 1 & $<0,1$ & 0,9 & $<0,1$ & $\mathrm{~V}^{8}$ \\
\hline Menticirrhus littoralis & 84 & 1,0 & 275,6 & 0,5 & $\mathrm{~V}^{8}$ \\
\hline Micropogonias furnieri & 65 & 0,8 & 566,2 & 1,1 & $\mathrm{~V}^{8}$ \\
\hline Stellifer rastrifer & 1 & $<0,1$ & 7,9 & $<0,1$ & $\mathrm{~V}^{8}$ \\
\hline Umbrina coroides & 4 & $<0,1$ & 13,3 & $<0,1$ & $\mathrm{~V}^{8}$ \\
\hline \multicolumn{6}{|l|}{ Serranidae } \\
\hline Mycteroperca sp. & 2 & $<0,1$ & 26,5 & 0,1 & \\
\hline \multicolumn{6}{|l|}{ Sparidae } \\
\hline Archosargus rhomboidalis & 18 & 0,2 & 192,7 & 0,4 & $\mathrm{~V}^{8}$ \\
\hline Diplodus argenteus & 176 & 2,1 & 315,8 & 0,6 & $\mathrm{~V}^{8}$ \\
\hline \multicolumn{6}{|l|}{ Synodontidae } \\
\hline Synodus foetens & 8 & 0,1 & 129,0 & 0,2 & $\mathrm{~V}^{8}$ \\
\hline \multicolumn{6}{|l|}{ Tetraodontidae } \\
\hline Sphoeroides testudineus & 3 & 0,0 & 40,7 & 0,1 & $\mathrm{R}^{8}$ \\
\hline Total & 8298 & 100 & 52902,4 & 100 & \\
\hline
\end{tabular}

Quanto à biomassa, foi obtido um total de $52.902,4 \mathrm{~g}$ e as espécies que mais contribuíram para tal foram M. liza (45,8\%), sendo a mais representativa e, juntamente com $M$. curema $(16,8 \%)$, E. argenteus (6,7\%) e $A$. brasiliensis $(5,8 \%)$, contribuíram com aproximadamente mais de $75 \%$ do total. As demais espécies pouco contribuíram para a biomassa total, sendo que 28 destas contribuíram com $0,1 \%$ ou menos do total (Tabela 1).

\section{Variações espaço-temporais}

A família Mugilidae, dominante na assembleia amostrada em termos numéricos, foi representada por M. curema e Mugil spp., cada uma representando aproximadamente $38 \%$ do total da família, seguidas de M. liza (24\%) e M. gaimardianus (0,3\%). Indivíduos desta família foram registrados nas quatro áreas e em todas as estações amostradas, com uma distribuição aproximadamente uniforme, mas com predominância numérica na área $\mathrm{M}$, principalmente na estação Out 89. Já a biomassa, que somou 33.862,2 g, teve maior participação de $M$. liza (71,6\%) e M. curema (26,3\%), enquanto Mugil spp. e M. gaimardianus juntas representaram apenas $2,1 \%$. Maiores valores médios de biomassa foram observados nas áreas S (Prim 88) e M (Ver 88).

Considerando o restante da ictiofauna (exceto Mugilidae), os verões (87 e 88), estações com maiores índices pluviométricos médios, foram as que apresentaram maiores valores médios dos descritores da comunidade ( $\mathrm{n}, \mathrm{S}$ e biomassa) nas quatro áreas amostradas (Figura 2a, 2b, 2c e 2d). O teste global de PERMANOVA revelou diferenças significativas entre as estações ( $n$, biomassa, riqueza de espécies e H’Loge), entre as áreas (n e riqueza de espécies) e nas interações entre os fatores estação e área (n e biomassa) (Tabela 2). 
FIGURA 2: Variações espaço-temporais nos descritores da assembleia de peixes amostrada na Lagoa da Conceição, SC, entre 1987 e 1989 $($ Prim $=$ Primavera; Ver $=$ Verão; Out $=$ Outono; Inv = Inverno $)$.

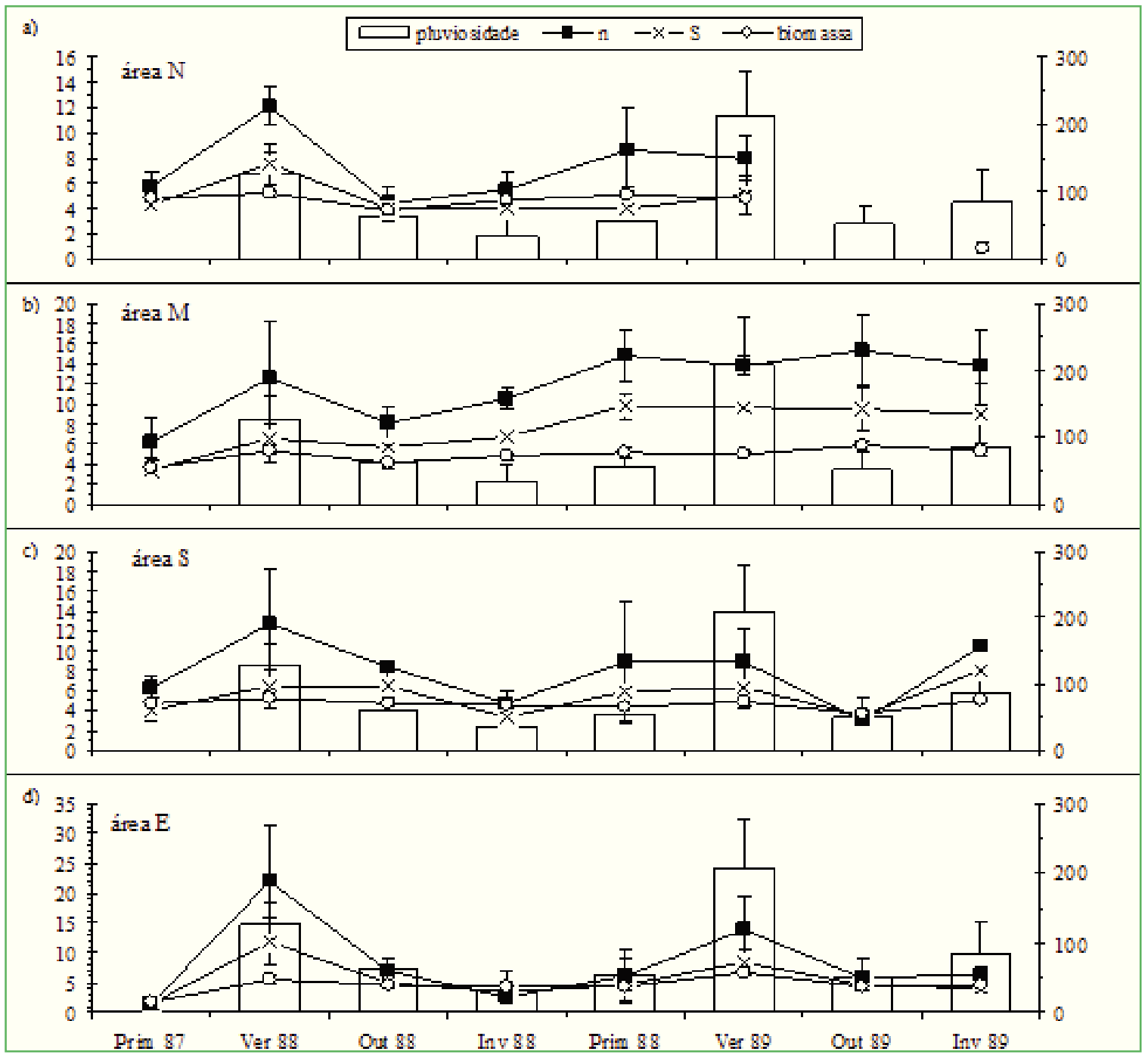

TABELA 2: Valores da análise de variância permutacional multivariada (PERMANOVA) (teste global) do número de indivíduos $(\mathrm{n})$, biomassa (ambos transformados em $\log (\mathrm{x}+1)$ ), riqueza de espécies $(\mathrm{S})$, Diversidade de Shannon-Wiener (H'loge) e Equitatividade de Pielou (J'), com base na similaridade de Bray-Curtis, para análises de variações temporais e espaciais da assembleia dos peixes coletados na Lagoa da Conceição, SC durante os anos de 1987, 1988 e $1989(* \mathrm{p}<0,05 ; * * \mathrm{p}<0,01 ; * * * \mathrm{p}<0,001 ; \mathrm{NS}=$ não significativo, $\mathrm{p}>0,05)$.

\begin{tabular}{lccccccccccc}
\hline & & $\mathbf{n}$ & \multicolumn{2}{c}{ biomassa } & \multicolumn{2}{c}{$\mathbf{S}$} & \multicolumn{2}{c}{ H'Log $_{\mathrm{e}}$} & \multicolumn{2}{c}{$\mathbf{J}^{\prime}$} \\
\hline & $\mathbf{F}$ & $\mathbf{p}$ & $\mathbf{F}$ & $\mathbf{p}$ & $\mathbf{F}$ & $\mathbf{p}$ & $\mathbf{F}$ & $\mathbf{p}$ & $\mathbf{F}$ & $\mathbf{p}$ \\
\hline estação & 4,1738 & $0,0002^{* * *}$ & 2,6398 & $0,019^{*}$ & 3,8641 & $0,0002^{* * *}$ & 3,2579 & $0,0049^{*}$ & 1,1769 & $0,3293^{\mathrm{NS}}$ \\
área & 6,4435 & $0,0001^{* * *}$ & 2,1132 & $0,08^{\mathrm{NS}}$ & 6,5252 & $0,0001^{* * *}$ & 1,7981 & $0,1428^{\mathrm{NS}}$ & 0,55884 & $0,6392^{\mathrm{NS}}$ \\
est. x área & 1,6958 & $0,0195^{*}$ & 1,9868 & $0,029^{*}$ & 1,444 & $0,0848^{\mathrm{NS}}$ & 0,86635 & $0,6263^{\mathrm{NS}}$ & 6,7657 & $0,3136^{\mathrm{NS}}$ \\
\hline
\end{tabular}


Apenas $\mathrm{n}$ apresentou valores médios significativamente diferentes na área N. Apenas Ver 88 foi significativamente maior que Prim 87 e Out e Inv 88 (Tabela 3). Apesar disso, houve uma tendência a se observar valores médios maiores dos três descritores nas estações mais chuvosas e quentes como Ver 88 e Ver 89 , com valores menores sendo observados nas estações menos chuvosas e mais frias (Figura 2a).

Já na área $\mathrm{M}$, diferenças significativas foram observadas nos três descritores, com tendência a menores valores ocorrerem na estação seca (Out/Inv) em detrimento da estação chuvosa, Ver (Figura 2b).
Valores médios de $\mathrm{n}$ foram significativamente menores nas estações mais frias (Out/Inv 88). A biomassa foi significativamente menor no Inv 88 , em relação ao Ver 88 , enquanto a riqueza de espécies foi menor na Prim 87 (Tabela 2).

Observou-se que as áreas S (Figura 2c) e E (Figura 2d) foram mais influenciadas pelas chuvas, apresentando maiores valores médios de $\mathrm{n}$, riqueza de espécies e biomassa nas estações mais chuvosas (Verões), tendendo a diminuir em Out/Inv de ambos os anos. Apesar disso, diferenças estacionais significativas não foram observadas nestas duas áreas (Tabela 3).

TABELA 3: Sumário dos resultados da análise de variância permutacional multivariada (PERMANOVA) da comparação das assembleias de peixes da Lagoa da Conceição, $\mathrm{SC}$ nas quatro áreas $(\mathrm{N}=$ Norte; $\mathrm{M}=$ Mediana; $\mathrm{S}=\mathrm{Sul} ; \mathrm{E}=$ externa) e nas oito estações $($ Prim $=$ Primavera; Ver $=$ Verão; Out $=$ Outono; Inv $=$ Inverno $)(* p<0,05 ; * * p<0,01)$.

\begin{tabular}{|c|c|c|c|c|c|c|c|c|c|c|c|c|}
\hline & \multicolumn{4}{|c|}{$\mathbf{n}$} & \multicolumn{4}{|c|}{ Biomassa } & \multicolumn{4}{|c|}{ Riqueza de espécies } \\
\hline & $\mathbf{N}$ & M & $\mathbf{S}$ & $\mathbf{E}$ & $\mathbf{N}$ & M & $\mathbf{S}$ & $\mathbf{E}$ & $\mathbf{N}$ & M & $\mathbf{S}$ & $\mathbf{E}$ \\
\hline Prim 87 x Ver 88 & $3,04 *$ & $1,98^{*}$ & 1,10 & 2,17 & 0,86 & 1,24 & 0,41 & 4,33 & 1,92 & $2,12 *$ & 1,34 & 3,09 \\
\hline Prim $87 \times$ Out 88 & 0,96 & 1,15 & 1,65 & 2,09 & 1,27 & 0,82 & 0,10 & 4,99 & 0,68 & 1,70 & 1,34 & 3,21 \\
\hline Prim 87 x Inv 88 & 0,09 & $1,99^{*}$ & 0,82 & 0,90 & 0,16 & 1,26 & 0,54 & 4,09 & 0,32 & $2,36^{*}$ & 0,52 & 1,85 \\
\hline Prim 87 x Prim 88 & 0,97 & $1,91 *$ & 0,54 & 0,70 & 0,32 & 1,07 & 0,45 & 2,44 & 0,30 & $2,42 *$ & 0,51 & 1,16 \\
\hline Prim 87 x Ver 89 & 1,11 & $2,48 *$ & 0,54 & 2,04 & 0,31 & 1,33 & 0,19 & 6,97 & 0,41 & 3,19 & 0,66 & 2,71 \\
\hline Prim 87 x Out 89 & & 1,71 & 2,87 & 1,91 & & 1,22 & 2,04 & 4,42 & & $2,01 * *$ & 0,39 & 2,81 \\
\hline Prim 87 x Inv 89 & 3,44 & 1,30 & 1,44 & 1,18 & 5,77 & 0,77 & 1,12 & 3,03 & 4,53 & 1,51 & 1,56 & 1,58 \\
\hline Ver $88 \times$ Out 88 & $2,80 *$ & 2,36 & 0,67 & 2,02 & 2,23 & 2,28 & 0,36 & 1,10 & 1,45 & 1,87 & 0,00 & 2,21 \\
\hline Ver $88 \times$ Inv 88 & $2,73 *$ & 2,06 & 1,49 & 2,87 & 1,10 & $2,58^{*}$ & 0,74 & 1,32 & 1,86 & 1,73 & 1,83 & 2,71 \\
\hline Ver $88 \times$ Prim 88 & 1,09 & 0,47 & 0,54 & 1,09 & 0,36 & 1,12 & 0,51 & 0,75 & 1,48 & 0,41 & 0,41 & 1,29 \\
\hline Ver 88 x Ver 89 & 1,84 & 0,92 & 0,49 & 0,68 & 1,02 & 1,93 & 0,26 & 1,51 & 1,08 & 0,66 & 0,44 & 0,71 \\
\hline Ver $88 \times$ Out 89 & & 0,35 & 2,24 & 2,27 & & 0,13 & 1,40 & 1,17 & & 0,36 & 2,08 & 2,34 \\
\hline Ver 88 x Inv 89 & 6,44 & 0,60 & 0,26 & 1,27 & 16,59 & 1,33 & 0,09 & 0,44 & 3,04 & 0,49 & 0,58 & 1,58 \\
\hline Out 88 x Inv 88 & 0,76 & 1,59 & 1,57 & 2,33 & 1,01 & 1,17 & 0,56 & 0,76 & 0,54 & 1,16 & 1,83 & 1,48 \\
\hline Out $88 \times$ Prim 88 & 1,25 & 2,23 & 0,64 & 0,79 & 1,26 & 1,29 & 0,45 & 0,33 & 0,40 & 2,81 & 0,41 & 0,61 \\
\hline Out $88 \times$ xer 89 & 1,54 & $3,22 * *$ & 0,47 & 1,00 & 1,55 & 1,58 & 0,15 & 3,76 & 0,53 & $4,67^{*}$ & 0,44 & 1,07 \\
\hline Out $88 \times$ Out 89 & & 1,73 & 5,55 & 1,17 & & 2,06 & 1,91 & 0,43 & & 1,57 & 2,08 & 1,00 \\
\hline Out $88 \times$ Inv 89 & 1,82 & 1,59 & 2,00 & 0,48 & 4,44 & 1,12 & 0,56 & 2,25 & 1,44 & 1,55 & 0,58 & \\
\hline Inv $88 \times$ Prim 88 & 0,86 & 1,67 & 0,61 & 0,73 & 0,44 & 0,72 & 0,44 & 0,14 & 0,07 & 2,25 & 0,88 & 0,42 \\
\hline Inv 88 x Ver 89 & 1,03 & $2,32 *$ & 0,94 & 2,32 & 0,49 & 0,71 & 0,51 & 3,37 & 0,50 & $3,46^{*}$ & 1,02 & 1,97 \\
\hline Inv $88 \times$ Out 89 & & 1,35 & 0,94 & 1,96 & & 2,06 & 1,66 & 0,32 & & 1,29 & 0,31 & 1,07 \\
\hline Inv $88 \times$ x Inv 89 & 2,84 & 1,08 & 1,23 & 1,43 & 5,58 & 0,88 & 0,91 & 0,60 & 4,01 & 1,02 & 1,85 & 0,50 \\
\hline Prim $88 \times$ Ver 89 & 0,23 & 0,33 & 0,29 & 0,91 & 0,21 & 0,23 & 0,53 & 1,98 & 0,39 & 0,23 & 0,17 & 0,97 \\
\hline Prim $88 \times$ Out 89 & & 0,26 & 0,91 & 0,73 & & 0,90 & 0,38 & 0,22 & & 0,38 & 0,74 & 0,49 \\
\hline Prim 88 x Inv 89 & 2,35 & 0,28 & 0,41 & 0,45 & 5,46 & 0,17 & 0,35 & 0,28 & 2,66 & 0,50 & 0,48 & 0,26 \\
\hline Ver 89 x Out 89 & & 0,59 & 1,52 & 1,25 & & 1,50 & 1,49 & 3,56 & & 0,66 & 0,85 & 1,27 \\
\hline Ver 89 x Inv 89 & 4,13 & 0,32 & 0,38 & 0,73 & 15,52 & 0,41 & 0,23 & 2,12 & 2,21 & 0,86 & 0,46 & 1,00 \\
\hline Out 89 x Inv 89 & & 0,33 & 3,69 & 0,49 & & 0,74 & 1,59 & 0,64 & & 0,23 & 3,17 & 0,58 \\
\hline
\end{tabular}


$\mathrm{Na}$ análise canônica de coordenadas principais (CAP), em relação às estações do ano (Figura 3), observou-se separação das amostras de outono e de verão, sendo que as amostras de primavera e inverno estiveram agrupadas no centro do gráfico, associadas ao eixo 1. Em relação às espécies, $P$. saltatrix e $A$. rhomboidalis estiveram correlacionadas com as amostras de outono e $A$. brasiliensis, E. argenteus, H. unifasciatus e E. melanopterus com as amostras de verão. O táxon Poecilia sp. esteve correlacionado com as amostras da primavera.

Em relação às áreas (Figura 4), ficou evidente a separação das amostras da área externa em relação às demais áreas, associadas ao eixo 1. Todavia, observou-se também uma separação das amostras das áreas mediana, norte e sul associadas ao eixo 2, com as amostras da área mediana e da área sul nos extremos. As espécies $E$. argenteus, $P$. saltatrix, $D$. argenteus e $M$. littoralis estiveram correlacionadas com as amostras da área externa e G. genidens e M. furnieri com as amostras da área sul. As espécies E. brasilianus e G. brasiliensis estiveram correlacionadas tanto com as amostras da área norte quanto com as amostras da área sul.

FIGURA 3: Resultado da análise canônica de coordenadas principais (CAP) para as estações do ano, com as espécies correlacionadas com as amostras coletadas na Lagoa da Conceição. A correlação canônica dos dois eixos obtida pela análise foi de $\delta_{1}=0,89$ e $\delta_{2}=0,70$

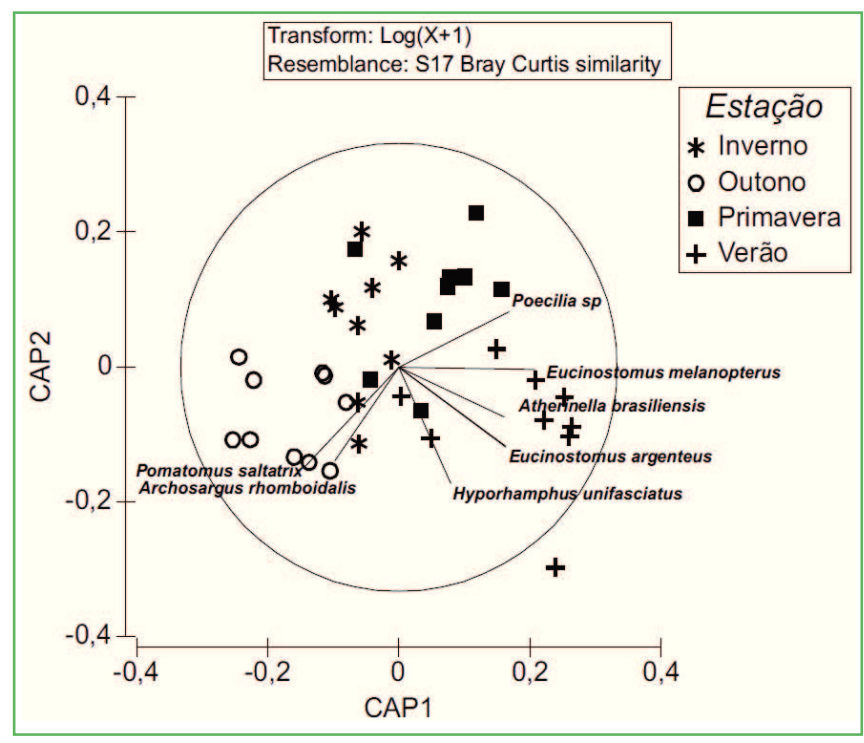

Revista Biotemas, 28 (3), setembro de 2015
FIGURA 4: Resultado da análise canônica de coordenadas principais (CAP) para as áreas, com as espécies correlacionadas com as amostras coletadas na Lagoa da Conceição. A correlação canônica dos dois eixos obtida pela análise foi de $\delta_{1}=0,95$ e $\delta_{2}=0,84$.

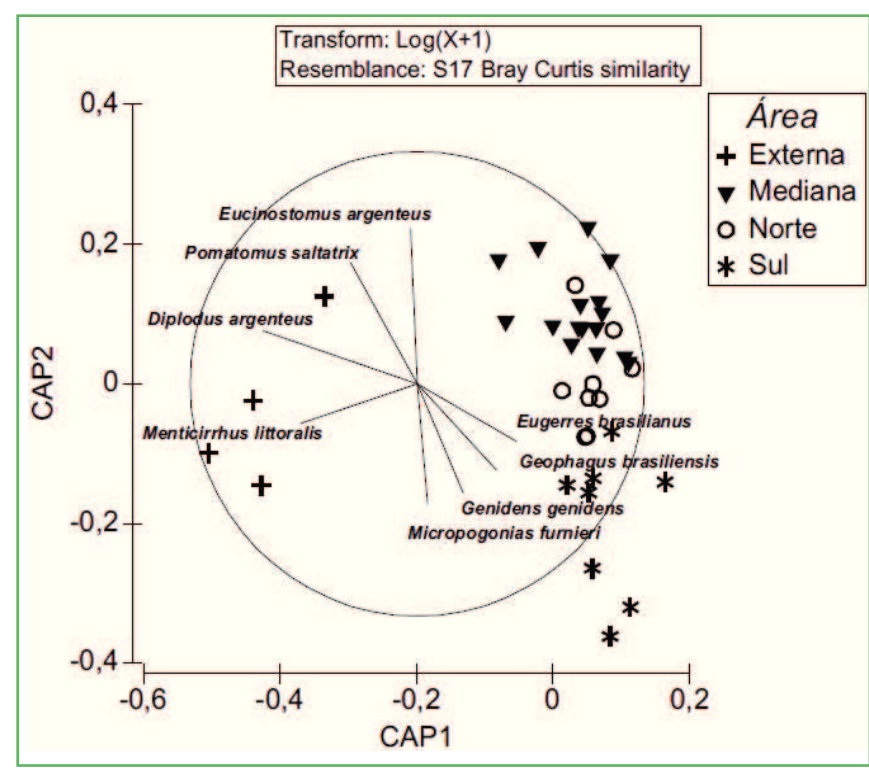

\section{Discussão}

Quanto à composição, a ictiofauna da Lagoa da Conceição definida como um ambiente lagunar costeiro foi semelhante à de regiões estuarinas, ambas marcadas pela dominância de poucas espécies devida principalmente ao fato de serem regiões de alterações nas variáveis físicas e químicas (DAY et al., 1989). De acordo com Yañez-Arancibia (1986), em áreas tropicais e subtropicais de ambientes costeiros estuarinos e lagunares há predominância de famílias como, por exemplo, Ariidae, Clupeidae, Engraulidae, Sciaenidae, Poeciliidae, Mugilidae, Gerreidae, Belonidae, Gobiidae. Este fato, observado no presente estudo demonstra a semelhança ecológica entre os dois ambientes, uma vez que ambos são considerados transicionais entre ambientes marinhos e dulcícolas e apresentam composição de espécies similar.

Na região estudada, as famílias que apresentaram o maior número de espécies foram Carangidae, Gerreidae, Sciaenidae, Gobiidae e Engraulidae, semelhantes a outros estudos realizados em diferentes ambientes estuarinos subtropicais no complexo estuarino de Paranaguá (CEP) no estado do Paraná (VENDEL et al., 
2003; SPACH et al., 2004). Estas famílias são citadas como predominantes para as regiões tropicais (YAÑEZARANCIBIA, 1986) e foram observadas também por Araújo (1998) e Pessanha et al. (2000) em lagunas costeiras tropicais na Baía de Sepetiba, RJ e apesar da Lagoa da Conceição, SC e do CEP estarem localizados na região subtropical as famílias que apresentaram maior diversidade encontram-se entre aquelas apontadas como as mais diversas para a região tropical.

Já em relação ao número de indivíduos na área estudada, as famílias Mugilidae, Gerreidae e Atherinopsidae foram as mais representativas. A família Mugilidae representou mais de $50 \%$ da captura total, e este fato pode estar relacionado com a presença de áreas com grande disponibilidade de alimentos, devido a alta produtividade primária e a maior quantidade de matéria orgânica acumulada, devido a baixa velocidade das correntes e, consequente maior tempo de renovação da água, são fatores existentes em ambientes lagunares (MITHTHAPALA, 2013) que podem influenciar na distribuição dos indivíduos juvenis desta família (VIEIRA, 1991). As famílias Atherinopsidae e Gerreidae são comuns em áreas rasas de ambientes subtropicais (FALCÃO et al., 2008; CORTELLETE et al., 2009), também foram encontradas por Pessanha et al. (2000) em lagunas costeiras da Baía de Sepetiba (RJ) e, segundo Ramos e Vieira (2001), a dominância numérica dessas famílias é relevante para o Atlântico oeste subtropical.

O maior número de espécies visitantes que o de espécies residentes já é um fato observado em ambientes estuarinos (HAEDRICH, 1983), sobretudo em regiões subtropicais (BLABER, 2000). O mesmo padrão também foi constatado para a Lagoa da Conceição, uma vez que a predominância foi de espécies visitantes e a grande maioria como juvenis, sugerindo mais uma vez, a similaridade ecológica entre laguna e estuários em termos de busca de alimento (BLABER; BLABER, 1980) e refúgio contra predação (PATERSON; WHITFIELD, 2000).

Em relação à importância comercial das espécies coletadas durante o estudo, entre as 14 espécies mais abundantes em biomassa $(+80 \%)$, seis destas são comercialmente relevantes, dentre estas as tainhas $M$. liza e M. curema, enchova (P. saltatrix), sardinha cascuda
(H. clupeola), xaréu (Caranx latus) e a corvina ( $M$. furnieri). Todas estas espécies por serem visitantes, mais uma vez reforçam a importância ecológica do ambiente da laguna costeira como área de alimentação, refúgio e recrutamento de espécies, já que a maioria destas se encontra em fases de recrutamento dentro da Lagoa da Conceição.

Mesmo que a maior parte das espécies capturadas neste estudo não tenha valor econômico, está claro o papel ecológico da área estudada para as diversas espécies de peixe que, em grande parte servem de alimento para peixes de importância econômica (ROBERTSON; DUKE, 1990), bem como tem a função de exportar a matéria e sedimentos orgânicos da laguna, para regiões adjacentes e ser incorporada nas redes tróficas fora da costa (LEBRETON et al., 2011).

\section{Referências}

AKIN, S.; BUHAN, E.; WINEMILLER, K. O.; YILMAZ, H. Fish assemblage structure of Koycegiz Lagoon estuary, Turkey: spatial and temporal distribution patterns in relation to environmental variation. Estuarine Coastal and Shelf Science, London, v. 64, p. 671-684, 2005.

AMEZCUA, F.; AMEZCUA-LINARES, F. Seasonal changes of fish assemblages in a subtropical Lagoon in the SE Gulf of California. The Scientific World Journal, Nars City, v. 2014, p. $1-15,2014$.

ANDERSON, M. J.; GORLEY, R. N.; ClARKE, K. R. PERMANOVA. PRIMER: guide to software and statistical methods. Plymouth: PRIMER-E Ltd., 2008. 214 p.

ANDREATA, J. V.; SAAD, A. M.; BARBIÉRI, L. R. R. Associação e distribuição das espécies de peixes na Laguna de Marapendi, Rio de Janeiro, Brasil. Atlântica, Rio Grande, v. 12, n. 1, p. 5-17, 1989. ARAÚJO, F. G. Adaptação do índice de integridade biótica usando a comunidade de peixes para o rio Paraíba do Sul. Revista Brasileira de Biologia, São Carlos, v. 58, n. 4, p. 547-558, 1998.

BARLETTA, M.; AMARAL, C. S.; CORRÊA, M. F. M.; GUEBERT, F.; DANTAS, D. V.; LORENZI, L.; SAINT-PAUL, U. Factors affecting seasonal variations in demersal fish assemblages at an ecocline in a tropical-subtropical estuary. Journal of Fish Biology, Perthshire, v. 73, p. 1314-1336, 2008.

BLABER, S. J. M. Tropical estuarine fishes, ecology, exploitation and conservation. Fish and Aquatic Resource Series 7. Malden: Blackwell Science, 2000. 372 p.

BLABER, S. J. M; BLABER, T. G. Factors affecting the distributions of juvenile estuarine and inshore fish. Journal of Fish Biology, Perthshire, v. 17, n. 2, p.143-162, 1980.

CHAVES, P. T. C.; OTTO, G. Aspectos biológicos de Diapterus rhombeus (Cuvier) (Teleostei, Gerreidae) na Baía de Guaratuba, 
Paraná, Brasil. Revista Brasileira de Zoologia, Curitiba, v. 15, n. 2, p. 289-295, 1998.

CHAVES, P. T. C.; VENDEL, A. L. Reprodução de Stellifer rastrifer (Jordan) (Teleostei, Sciaenidae) na Baía de Guaratuba, Paraná, Brasil. Revista Brasileira de Zoologia, Curitiba, v. 14, n. 1, p. 81-89, 1997.

CONSTANZA, R.; DARGE, R.; DEGROOT, R.; FARBER, S.; GRASSO, M.; HANNON, B.; LIMBURG, K.; NAEEM, S.; ONEILL, R. V.; PARUELO, J.; RASKIN, R. G.; SUTTON, P.; VANDENBELT, M. The value of the world's ecosystem services and natural capital. Nature, London, v. 387, p. 253-260, 1997.

CORTEllete, G. M.; GODEFROID, R. S.; SILVA, A. L. C.; CATTANII, A. P.; DAROS, F. A.; SPACH, H. L. Peixes da área de deposição de material dragado na Baía de Antonina, Paraná, Brasil. Cadernos da Escola de Saúde, Curitiba, v. 2, p. 1-19, 2009.

CROMWELL, J. E. Barrier coast distribution: a world survey. In: NATIONAL COASTAL AND SHALLOW WATER RESEARCH CONFERENCE, 2, 1971, Baton Rouge. Abstract... Baton Rouge, 1971. p. 50.

DAY, J. W.; HALL, C. A. S.; KEMP, W. M.; YAÑEZ-ARANCIBIA, A. Estuarine Ecology. New York: John Wiley \& Sons, 1989. 558 p.

ELLIOTT, M.; MCLUSKY, D. S. The need for definitions in understanding estuaries. Estuarine, Coastal and Shelf Science, London, v. 55, n. 6, p. 815-827, 2002.

FALCÃO, M. G.; PICHLER, H. A.; FÉLIX, F. C.; SPACH, H. L.; BARRIL, M. E.; ARAÚJO, K. C. B.; GODEFROID, R. S. A ictiofauna como indicador de qualidade ambiental em planícies de maré do complexo estuarino de Paranaguá, Brasil. Cadernos da Escola de Saúde, Curitiba, v. 1, p. 1-16, 2008.

FIGUEIREDO, J. L.; MENEZES, N. Manual de peixes marinhos do sudeste do Brasil. II. Teleostei (1). São Paulo: Museu de Zoologia da USP, 1978. $110 \mathrm{p}$.

FIGUEIREDO, J. L.; MENEZES, N. Manual de peixes marinhos do sudeste do Brasil. III. Teleostei (2). São Paulo: Museu de Zoologia da USP, 1980. 90 p.

FIGUEIREDO, J. L.; MENEZES, N. Manual de peixes marinhos do sudeste do Brasil. VI. Teleostei (5). São Paulo: Museu de Zoologia da USP, 2000. 116 p.

FROESE, R.; PAULY, D. Fishbase. Disponível em: <www. fishbase.com>. Acesso em: 21 jan. 2014.

FROTA, L. O. R.; CARAMASCHI, E. P. Aberturas artificiais de barra da lagoa Imboassica e seus efeitos sobre a fauna de peixes. In: ESTEVES, F. A. (Ed.). Ecologia das lagoas costeiras do Parque Nacional da Restinga de Jurubatiba e do município de Macaé (RJ). Rio de Janeiro: UFRJ/Departamento de Ecologia, 1998. p. 327-350.

GARCIA, A. M.; VIEIRA, J. P. O aumento da diversidade de peixes no estuário da Lagoa dos Patos durante o episódio El Niño 19971998. Atlântica, Rio Grande, v. 23, p. 133-152, 2001.

GREENWOOD, M. F. D.; HILL, A. S. Temporal, spatial and tidal influences on benthic and demersal fish abundance in the Forth estuary. Estuarine Coastal and Shelf Science, London, v. 58, p. 211-225, 2003.

HAEDRICH, R. L. Estuarine fishes. In: KETCHUM, B. H. (Ed.). Estuaries and enclosed seas. New York: Elsevier Scientific, 1983. p. 183-207.
JAUREGUIZAR, A. J.; MENNI, R.; GUERRERO, R.; MIANZAN, H.; LASTA, C. Environmental factors structuring fish communities of the Rio de la Plata estuary. Fisheries Research, Amsterdam, v. 66 , p. $195-211,2004$

KENNISH, M. J.; PEARL, H. W. Coastal lagoons: critical habitats of environmental change. Boca Raton: CRC Press, 2010. 387 p.

KJERVFE, B. Coastal lagoons. In: KJERFVE, B. (Ed.) Coastal lagoon processes. Amsterdam: Elsevier Oceanography Series, 1994. p. $1-8$.

KLINGEBIEL, A.; SIERRA DE LEDO, B. Etude preliminaire des mareé la Lagoa da Conceição, 116 de Santa Catarina. In: COLÓQUIO FRANCO-BRASILEIRO DE MANEJO COSTEIRO DA ILHA DE SANTA CATARINA, 1, 1997, Florianópolis. Atas... Florianópolis, 1997. p. 129-140.

KNOPPERS, B. Aquatic primary production in coastal lagoons. In: KJERFVE, B. (Ed.). Costal Lagoon Processes. Amsterdam: Elsevier Science, 1994. p. 1-8.

KNOPPERS, B. A.; OPITZ, S. S.; DE SOUZA, M. P.; MIGUEZ, C. F. The spatial distribution of particulate organic matter and some physical and chemical water properties in Conceição Lagoon, Santa Catarina, Brazil. Arquivos de Biologia e Tecnologia, Curitiba, v. 27, n. 1, p. 59-77, 1984.

KUPSCHUS, S.; TREMAIN, D. Associations between fish assemblages and environmental factors in nearshore habitats of a subtropical estuary. Journal of Fish Biology, Perthshire, v. 58, n. 5, p. 1383-1403, 2001.

LEBRETON, B.; RICHARD, P.; PARLIER, E. P.; GUILLOU, G.; BLANCHARD, G. F. Trophic ecology of mullets during their spring migration in a European saltmarsh: a stable isotope study. Estuarine, Coastal and Shelf Science, London, v. 91, n. 4, p. 502$510,2011$.

LIMA, N. R. W.; BIZERRIL, C. R. S. F.; SUZUKI, M. S; CANIÇALI, M. R.; FERREIRA, A. G.; GOMES, M. A. A.; ASSUMPÇÃO, J.; PAES, M.; FARIA, V. Impacto da abertura de barra sobre a ictiofauna da Lagoa de Iquipari, norte do estado do Rio de Janeiro. Revista Bios, Belo Horizonte, v. 9, n. 9, p. 73-82, 2001.

LISBOA, L. K. L.; TEIVE, L. F.; PETRUCIO, M. M. Lagoa da Conceição: uma revisão da disponibilidade de dados ecológicos visando o direcionamento de novas pesquisas no ecossistema. Biotemas, Florianópolis, v. 21, n. 1, p. 139-146, 2008.

LOEBMANN, D.; VIEIRA, J. P. Distribuição espacial e abundância das assembleias de peixes no Parque Nacional da Lagoa do Peixe, Rio Grande do Sul, Brasil. Revista Brasileira de Zoologia, Curitiba, v. 22, n. 3, p. 667-675, 2005.

MACI, S., BASSET, A. Composition, structural characteristics and temporal patterns of fish assemblages in non-tidal Mediterranean lagoons: A case study. Estuarine, Coastal and Shelf Science, London, v. 83, n. 4, p. 602-612, 2009.

MARQUES, A. B. Biologia reprodutiva do bagre Genidens genidens (Valenciennes, 1839) na lagoa do Açu, Norte Fluminense, RJ. 2005. 86 f. Dissertação (Mestrado em Ecologia) Universidade Estadual do Norte Fluminense, Rio de Janeiro. 2005.

MATSUURA, L. Y. Brazilian sardine (Sardinella brasiliensis) spawning in the southeast Brazilian bight over the period 19761993. Revista Brasileira de Oceanografia, São Paulo, v. 46, n. 1, p. 33-43, 1998. 
MENEZES, N.; FIGUEIREDO, J. L. Manual de peixes marinhos do sudeste do Brasil. IV. Teleostei (3). São Paulo: Museu de Zoologia da USP, 1980. $90 \mathrm{p}$.

MITHTHAPALA, S. Lagoons and estuaries. Coastal Ecosystems Series (Vol. 4). Colombo: IUCN Sri Lanka Country Office, 2013. $73 \mathrm{p}$.

MPB - MPB SANEAMENTO S/C LTDA. Contorno de Florianópolis. Relatório de impacto sobre o meio ambiente (RIMA) referente à implantação do contorno de Florianópolis/SC. 2011. Florianópolis: MPB SANEAMENTO S/C LTDA, 2011. 223 p.

MUEHE, D.; GOMES JR., F. C. Batimetria e algumas considerações sobre a evolução geológica da Lagoa da Conceição, Ilha de Santa Catarina. In: LEDO, B. S.; SORIANO-SERRA, E. J. (Ed.). O ecossistema da Lagoa da Conceição. Florianópolis: NEMAR, 1999. p. 15-24.

ODEBRECHT, C.; CARUSO JR., F. G. Hidrografia e matéria particulada em suspensão na Lagoa da Conceição, Ilha de Santa Catarina, SC, Brasil. Atlântica, Rio Grande, v. 9, n. 1, p. 83-104, 1987.

PASSOS, A. C.; CONTENTE, R. F.; ABBATEPAUlO, F. V.; SPACH, H. L.; VILAR, R. C. C.; JOYEUX, J. C.; CARTAGENA, B. F. C.; FAVARO, L. F. Analysis of fish assemblages in seconds along a salinity gradient based on species, families and functional groups. Brazilian Journal of Oceanography, São Paulo, v. 61, n. 4, p. 251-264, 2013.

PATERSON, A. W.; WHITFIELD, A. K. The ichthyofauna associated with an intertidal creek and adjacent eelgrass beds in the Kariega Estuary, South Africa. Environmental Biology of Fishes, New York, v. 58, n. 2, p. 145-156, 2000.

PÉREZ-RUZAFA, A.; GARCIA-CHARTON, J. A.; BARCALA, E.; MARCOS, C. Changes in benthic fish assemblages as a consequence of coastal works in a coastal lagoon: the Mar Menor (Spain, Western Mediterranean). Marine Pollution Bulletin, Amsterdam, v. 53, n. 1-4, p. 107-120, 2006.

PESSANHA, A. L. M.; ARAÚJO, F. G.; AZEVEDO, M. C. C.; GOMES, I. D. Variações temporais e espaciais na composição e estrutura da comunidade de peixes jovens da baía de Sepetiba, Rio de Janeiro. Revista Brasileira de Zoologia, Curitiba, v. 17, n. 1, p. 251-261, 2000.

RAMOS, L. A.; VIEIRA, J. P. Composição específica e abundância de peixes de zonas rasa dos cinco estuários do Rio Grande do Sul, Brasil. Boletim do Instituto de Pesca, São Paulo, v. 27, n. 1, p.109-121, 2001.

ROBERTSON, A. I.; DUKE, N. C. Recruitment, growth and residence time of fishes in a tropical Australian mangrove system. Estuarine, Coastal and Shelf Science, London, v. 31, n. 5, p. 723$743,1990$.
SIERRA DE LEDO, B. Subsídios ecológicos para um plano de gestão integrada na zona costeira da Ilha de Santa Catarina. In: COLÓQUIO FRANCO-BRASILEIRO DE MANEJO COSTEIRO DA ILHA DE SANTA CATARINA, 1, 1997, Florianópolis. Atas... Florianópolis, 1997. p. 9-28.

SIERRA DE LEDO, B.; GRÉ, J. C. R.; SORIANO-SIERRA, E. J. Fishery production anthropogenic and natural stress in Conceição Lagoon, Santa Catarina, Brazil. Florianópolis: NEMAR, 1985. 14 p.

SIERRA DE LEDO, B.; RIBEIRO, G. C.; CLEZAR, L.; HOSTIMSILVA, M. Padrões de ocorrência espacial e temporal de peixes mugilídeos jovens na Lagoa da Conceição, Ilha de Santa Catarina, Biotemas, Florianópolis, v. 6, n. 1, p. 133-146, 1993.

SPACH, H. L.; GODEFROID, R. S.; SANTOS, C.; SCHWARZ, J. R. R.; QUEIROZ, G. M. L. Temporal variation in fish assemblage composition on a tidal flat. Brazilian Journal of Oceanography, São Paulo, v. 52, n. 1, p. 47-58, 2004.

SUZUKI, M. S.; OVALLE, A. R. C.; PEREIRA, E. A. Effects of sand bar openings on some limnological variables in a hypertrofic tropical coastal laggon of Brasil. Hydrobiologia, Brussels, v. 368, n. 1-3, p. 111-122, 1998.

VENDEL, A. L.; BOUCHEREAU, J. L.; CHAVES, P. T. Environmental and subtidal fish assemblage relationships in two different Brazilian coastal estuaries. Brazilian Archives of Biology and Technology, Curitiba, v. 53, n. 6, p. 1393-1406, 2010.

VENDEL, A. L.; LOPES, S. G.; SANTOS, C.; SPACH, H. L. Fish assemblages in a tidal flat Brazil. Brazilian Archives of Biology and Technology, Curitiba, v. 46, n. 2, p. 233-242, 2003.

VERDIELL-CUBEDO, D.; OLIVA-PATERNA, F. J.; TORRALVA, M. Length-weight relationships for 22 fish species of the Mar Menor coastal lagoon (western Mediterranean Sea). Journal of Applied Ichthyology, Berlin, v. 22, n. 4, p. 293-294, 2006.

VIEIRA, J. P. Juvenile mullets (Pisces: Mugilidae) in the Estuary Lagoa dos Patos, RS, Brazil. Copeia, Lawrence, v. 1991, n. 2, p. 409-418, 1991.

VIEIRA, J. P.; MUSICK, J. A. Latitudinal patterns in diversity of fishes in warm-temperate and tropical estuarine waters of the Western Atlantic. Atlântica, Rio Grande, v. 15, p. 115-133, 1993.

VILAR, C. C.; SPACH, H. L.; JOYEUX, J. C. Spatial and temporal changes in the fish assemblage of a subtropical estuary in Brazil: environmental effects. Journal of the Marine Biological Association of the United Kingdom, Cambridge, v. 91, n. 3, p. 635-648, 2011.

WHITFIELD, A. K. Ichthyofaunal assemblages in estuaries: a South African case study. Reviews in Fish Biology and Fisheries, Washington, v. 9, p. 151-186, 1999.

YAÑEZ-ARANCIBIA, A. Ecología de la zona costera. Análisis de siete tópicos. Ciudad de México: AGT Editonal, 1986. 189 p. 